\title{
A TAF4 coactivator function for $E$ proteins that involves enhanced TFIID binding
}

\author{
Wei-Yi Chen, ${ }^{1}$ Jinsong Zhang, ${ }^{1}$ Huimin Geng, ${ }^{2}$ Zhimei Du, ${ }^{1}$ Tomoyoshi Nakadai, ${ }^{1}$ \\ and Robert G. Roeder ${ }^{1,3}$ \\ ${ }^{1}$ Laboratory of Biochemistry and Molecular Biology, The Rockefeller University, New York, New York 10065, USA; \\ ${ }^{2}$ Laboratory Medicine, University of California at San Francisco School of Medicine, San Francisco, California 94143, USA
}

The multisubunit TFIID plays a direct role in transcription initiation by binding to core promoter elements and directing preinitiation complex assembly. Although TFIID may also function as a coactivator through direct interactions with promoter-bound activators, mechanistic aspects of this poorly defined function remain unclear. Here, biochemical studies show a direct TFIID-E-protein interaction that (1) is mediated through interaction of a novel E-protein activation domain (activation domain 3 [AD3]) with the TAF homology (TAFH) domain of TAF4, (2) is critical for activation of a natural target gene by an E protein, and (3) mechanistically acts by enhancing TFIID binding to the core promoter. Complementary assays establish a gene-specific role for the TAFH domain in TFIID recruitment and activation of a large subset of genes in vivo. These results firmly establish TAF4 as a bona fide E-protein coactivator as well as a mechanism involving facilitated TFIID binding through direct interaction with an E-protein activation domain.

[Keywords: TAF4; TAFH; TFIID; transcription coactivator; E proteins; E2A; HEB]

Supplemental material is available for this article.

Received February 17, 2013; revised version accepted June 12, 2013.

Transcription of protein-coding genes by RNA polymerase II (Pol II) is a multistep process that involves activatordependent recruitment of chromatin remodeling/modifying coactivators (Roeder 2005; Li et al. 2007) followed by activator-dependent assembly/function of the transcription preinitiation complex (PIC) through additional coactivators (Naar et al. 2001; Roeder 2005). The latter group of coactivators includes the multisubunit Mediator and a variety of general and gene- or cell-specific coactivators. As bridging proteins, these coactivators transmit signals directly from activators to the basal transcription machinery, thereby stimulating the formation and/or function of the PIC. The PIC includes, in addition to Pol II, the general transcription factors (GTFs) TFIID, TFIIA, TFIIB, TFIIE, TFIIF, and TFIIH (Roeder 1996; Thomas and Chiang 2006). Of special interest here, TFIID is a multisubunit complex composed of the TATA box-binding protein (TBP) and 13-14 evolutionarily conserved TBPassociated factors (TAFs). TFIID is the primary core promoter recognition factor, through TBP binding to the TATA box and/or TAF interactions with other core promoter elements (Juven-Gershon et al. 2008), and thus plays the major role in directing PIC assembly, through interactions with other GTFs, for subsequent transcrip-

${ }^{3}$ Corresponding author

E-mail roeder@mail.rockefeller.edu

Article is online at http://www.genesdev.org/cgi/doi/10.1101/gad.216192.113. tion by Pol II (Burley and Roeder 1996; Roeder 1996). Consequently, TFIID binding to the core promoter has been widely believed to contribute a critical rate-limiting step at which enhancer-bound activators can regulate transcription activation, and genetic studies in yeast have provided support for this idea (Chatterjee and Struhl 1995).

Beyond the original studies (Hoffmann et al. 1990; Pugh and Tjian 1990), many in vitro transcription studies with naked DNA template have shown that whereas TBP suffices for basal transcription from TATA-containing promoters, robust activator-dependent transcription is dependent on TFIID, thus suggesting important coactivator functions for TAFs (Albright and Tjian 2000; Green 2000; Naar et al. 2001; Thomas and Chiang 2006). Although TAFs in the TFIID complex play important roles in modulating transcriptional activity, their specific functions and mechanisms of action are complex and, in many cases, poorly understood. On the one hand, specific metazoan TAFs can recognize and interact with nonTATA core promoter elements, such as Initiator (Inr) and downstream promoter elements (DPE), and show core promoter-specific functions (Juven-Gershon et al. 2008). On the other hand, and consistent with gene-selective coactivator functions through activator-TFIID interactions, a number of earlier studies showed direct interactions of diverse activators with specific isolated TAFs (references in Burley and Roeder 1996; Liu et al. 2009). 
Adding support to this idea, recent studies have shown direct interactions of several activators with specific TAFs in the more physiological context of intact TFIID complexes in both metazoans (Liu et al. 2009) and yeast (Garbett et al. 2007). Genetic analyses have provided further support for gene-selective TAF coactivator functions (Albright and Tjian 2000; Green 2000), including roles for specific activation domain-TAF interactions in TFIID recruitment (Garbett et al. 2007; Layer et al. 2010). However, these genetic studies are intrinsically limited due to their inability to establish direct effects of activators that, through broadly used activation domains, may recruit other factors (e.g., chromatin remodelers or other GTFs) that in turn facilitate or stabilize TFIID binding. In this regard, early in vitro studies with purified factors established direct qualitative and quantitative effects of activators on TFIID and/or TFIIA-TFIID binding to model promoters (Abmayr et al. 1988; Horikoshi et al. 1988; Lieberman and Berk 1994; Chi and Carey 1996) as well as a stabilizing effect of TFIID on activator binding (Sawadogo and Roeder 1985), although possible roles of specific activator-TAF interactions were not investigated.

TAF4, of interest here, plays an essential role in maintaining the stability and integrity of the TFIID complex (Wright et al. 2006). TAF4 is also the first individual TAF for which a coactivator function was reported and has been shown, as an isolated protein, to interact with many activators, including Sp1, CREB, RAR, CBF, NFAT, and c-Jun (references in Garbett et al. 2007). Although the mechanism by which TAF4 exerts its coactivator function is unknown, TAF4 and its paralog, TAF4b, contain an $\sim 100$ amino acid TAF homology (TAFH) domain that is also present in MTG/ETO family proteins and highly conserved in TAF4 homologs from Drosophila to humans (Zhang et al. 2004). Regarding TAF4 functions, disruption of the murine Taf4 gene leads to lethality on embryonic day 9.5, and microarray analyses of derived $\mathrm{Taf}^{-1-}$ murine embryonic fibroblasts (MEFs), which retain an intact TAF4b-containing TFIID complex, revealed deregulation of a large set of genes in the null cells (Mengus et al. 2005). These results suggest that TAF4- and TAF4b-containing TFIID complexes are differentially required for the transcription of certain genes. Indeed, TAF4b is selectively expressed in B-lineage and gonadal cells and required for the development and differentiation of ovarian granulocytes (Goodrich and Tjian 2010).

E proteins, which include E2A (E12 and E47), E2-2, and $\mathrm{HEB}$, are a ubiquitously expressed basic helix-loop-helix (bHLH) family of transcription factors that play important roles in embryonic patterning, cell fate determination and differentiation, regulation of cell proliferation, and programmed cell death (for review, see Quong et al. 2002; Kee 2009). E-protein dimers activate target genes, including $p 21$ (Prabhu et al. 1997), by binding to E boxes (canonical sequence CANNTG) at enhancer and/or promoter regions (Quong et al. 2002). In many cell types, E proteins also regulate gene expression in a tissuespecific manner by forming functional heterodimers with tissue-specific bHLH proteins such as MyoD. In addition to the bHLH domain, E proteins contain two conserved activation domains, termed activation domain 1 (AD1) and AD2 (Quong et al. 2002; Kee 2009). Previous studies have shown that the $\mathrm{N}$-terminal AD1 can directly interact with $\mathrm{p} 300 / \mathrm{CBP}$ histone acetyltransferase coactivators in gene activation (Bayly et al. 2004) or with corepressors AML1-ETO/ETO in gene silencing through a putative $\alpha$-helical domain (Zhang et al. 2004). AD2, also termed the loop-helix domain, is located midway between the AD1 and bHLH domains and has been reported to interact with CBP (Bayly et al. 2004) and SAGA (Massari et al. 1999) histone acetyltransferases.

Earlier studies from our laboratory showed that the E protein HEB interacts not only with p300/CBP (via AD1), but also with the TFIID complex (Zhang et al. 2004). However, the failure by us and others to observe an interaction of $\mathrm{AD} 1$ or $\mathrm{AD} 2$ with any component of the general transcription machinery (including TFIID) suggested the possible involvement of a distinct E-protein activation domain in the TFIID interaction. Here, in a major extension of previous studies, we first define a novel E-protein activation domain, designated AD3. We then employ rigorous biochemical analyses, involving TAF4-mutated forms of TFIID, to show that AD3, through interactions with the TAFH domain of TAF4, mediates a direct E-protein-TFIID interaction, is necessary for E-protein-dependent transcription from a natural target gene, and acts by stimulating the stable binding of TFIID to the natural promoter. Complementary cellbased assays with Taf4-null cells expressing ectopic wild-type versus TAFH-deleted forms of TAF4 confirm the physiological relevance of the AD3-TAFH interaction for selective E-protein target gene activation through TAF4-dependent recruitment of TFIID. In addition, genome-wide gene expression profiles also suggest that $\sim 400$ genes require the TAFH domain for TFIID recruitment and gene activation. Altogether, these studies provide an unprecedented documentation of a TAF coactivator function on a natural promoter as well as an underlying mechanism of action both in vitro and in vivo.

\section{Results}

E proteins interact directly with TFIID through the TAF4 subunit

We previously reported that corepressor ETO and the leukemogenic AML1-ETO fusion protein can repress E-protein-mediated transcription through a strong interaction between the ETO TAFH (eTAFH) domain and the $\mathrm{AD} 1$ domain of E proteins (Zhang et al. 2004). This result, the strong homology between the eTAFH domain and the TAFH domain (dTAFH) of TAF4 (Fig. 1A; Supplemental Fig. S1), and our demonstration of TFIID and p300/CBP (in unfractionated nuclear extract) binding to recombinant HEB (Zhang et al. 2004) suggested the possibility that TFIID might likewise interact with $\mathrm{E}$ proteins through TAF4 as part of an E-protein-mediated activation path- 


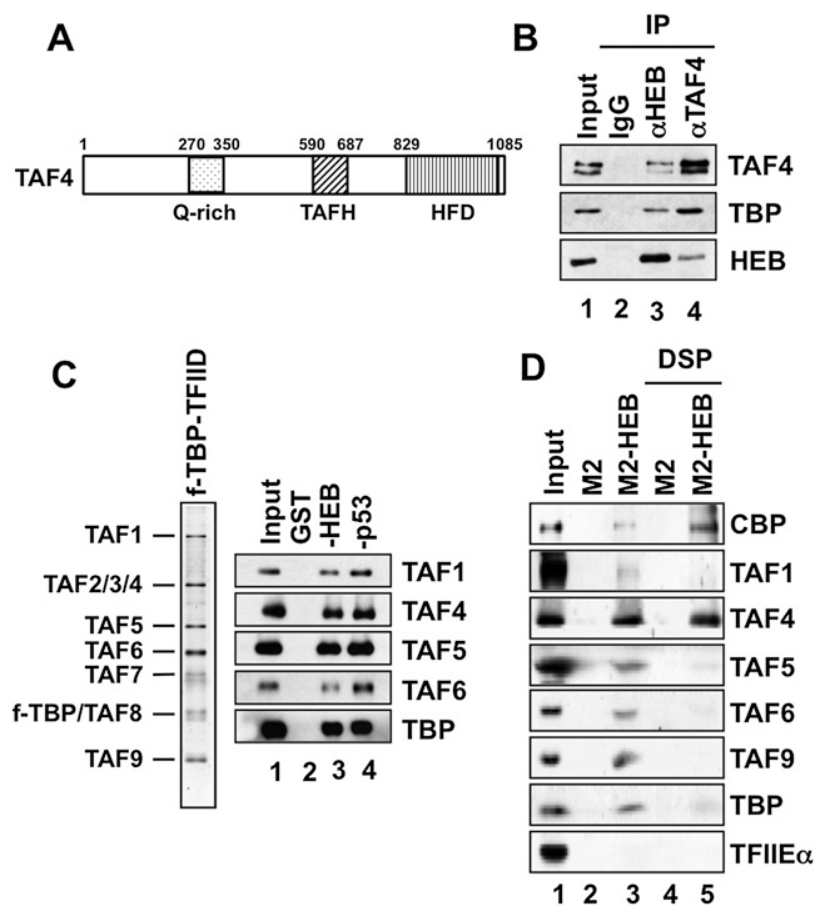

Figure 1. The TAF4 subunit in the TFIID complex is directly targeted by E proteins. (A) Schematic representation of human TAF4 protein. (Q-rich) Glutamine-rich; (HFD) histone fold domain. See also Supplemental Figure S1. (B) Coimmunoprecipitation assays showing an endogenous interaction between HEB and TAF4/TFIID. HeLa nuclear extract was immunoprecipitated (IP) with antibodies against HEB or TAF4. Bound proteins were visualized by immunoblotting with indicated antibodies. (C) GST pull-down assays showing the binding of the purified f-TBP-TFIID complex (silver stained, left) to GSTHEB or GST-p53 proteins. (D) Specific interaction of HEB with the TAF4 subunit in the context of the TFIID complex. HeLa nuclear extracts were incubated with f-HEB protein immobilized on anti-Flag M2 beads. Interacting complexes were washed with binding buffer (lanes 2,3) or treated with cross-linking reagent DSP and washed extensively with $8 \mathrm{M}$ urea buffer (lanes 4,5). After cross-link reversal by boiling in sample buffer, bound proteins were visualized by immunoblotting with the indicated antibodies. TFIIE $\alpha$ served as a control.

way. In approaching this question, we first established a natural intracellular association of E proteins and TFIID. Thus, reciprocal immunoprecipitation assays with antiHEB and anti-TAF4 antibodies each resulted in the coimmunoprecipitation of TFIID (monitored by TBP and TAF4 immunoblots) and HEB (Fig. 1B).

To establish that the intracellular TFIID-HEB association reflects a direct interaction rather than an association dependent on other unknown factors in the nuclear extract, we assessed the binding of affinity-purified TFIID (Fig. 1C, left) to purified GST-HEB and GST-p53 (as a positive control) fusion proteins. In clear confirmation of a direct interaction, TFIID bound strongly to both GST fusion proteins but not to GST (Fig. 1C, right). To determine whether, as expected, this interaction reflected an interaction of HEB with TAF4, we used dithiobis[succinimidyl propionate] (DSP), a reversible bifunctional cross-linker that cross-links proteins in close proximity, in a pulldown/cross-linking assay (details in the Supplemental Material). As shown in Figure 1D, CBP (which binds AD1 directly) (Zhang et al. 2004) and TAF4, but not TBP or other representative TAFs, were detected in the DSP/ urea-treated immune complex, whereas other subunits of TFIID were detected in the immune complex without DSP/urea treatment. These results clearly establish a direct interaction of HEB with TFIID through the TAF4 subunit.

The dTAFH domain of TAF4 interacts with a newly identified HEB transcriptional activation domain (AD3) that acts synergistically with $A D 1$ and $A D 2$

The above results, the homology between the TAFH domains, and the prior demonstration of an eTAFH interaction with HEB AD1 led us to predict that the TAF4 dTAFH domain would also interact with HEB through the AD1 domain. An initial analysis showed, as predicted, that HEB (predominant E protein in HeLa cells) and E2A (predominant E protein in Namalwa B cells) both interact with the isolated dTAFH domain (Fig. 2A). To test the interaction domains within HEB, we carried out binding assays with purified GST-fused dTAFH and eTAFH domains and in vitro-generated HEB fragments (Fig. 2B, left). Surprisingly, the dTAFH domain showed a strong selective interaction with an HEB fragment spanning residues 100-306, whereas the eTAFH domain interacted strongly (as expected) with a fragment (amino acids 1-99) containing AD1 and weakly with a fragment (amino acids 100-306) containing AD3 (Fig. 2B, right). Since the HEB region spanning residues 100-306 was specifically recognized by the dTAFH domain of TAF4/ TFIID and in light of subsequent functional data (below), it was designated AD3.

To establish the relevance of the dTAFH-HEB activation domain interactions (above) to TFIID-HEB activation domain interactions, we assessed the binding of endogenous TFIID in HeLa nuclear extracts (Fig. 2C) and purified TFIID (Fig. 2D) to GST fusion proteins containing HEB AD1, AD2, or AD3. Notably, endogenous TFIID (nuclear extract) and purified TFIID bound selectively to AD3, whereas CBP (nuclear extract) bound selectively to $\mathrm{AD} 1$. These results are in complete agreement with data (above) indicating that $\mathrm{AD} 3$ is recognized by the dTAFH domain and confirm AD3 as the HEB domain that specifically and directly interacts with the TFIID complex.

The ability of HEB AD3 to interact with the TAF4/ TFIID complex suggested that it is likely to possess a transcriptional function. To examine this possibility, the activation potentials of Gal4-HEB fragments were tested in a Gal4-based reporter assay involving transfection of 293T cells. Notably, the AD3 domain showed both an independent transcriptional activity that was greater than the previously reported $\mathrm{AD} 1$ and $\mathrm{AD} 2$ activities and a strong synergy with $\mathrm{AD} 1$ that was modestly enhanced by AD2 (Fig. 2E). A further investigation of this 
A

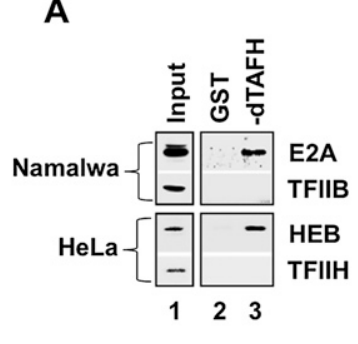

C

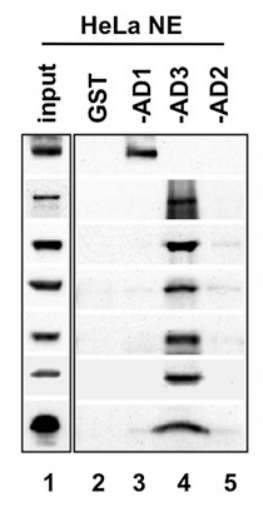

B

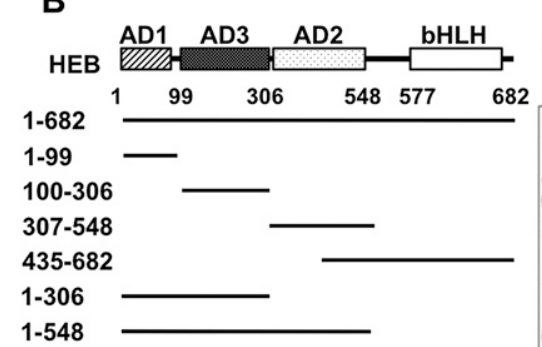

D

E

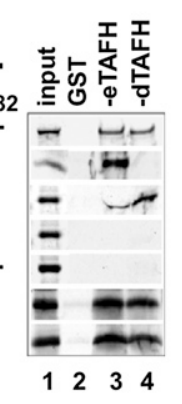

f-TBP-TFIID

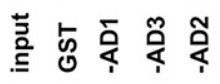

CBP

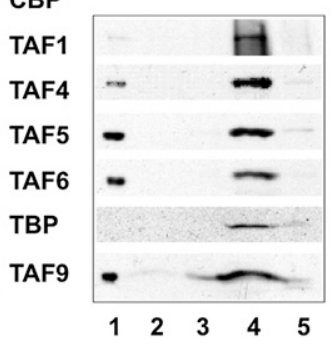

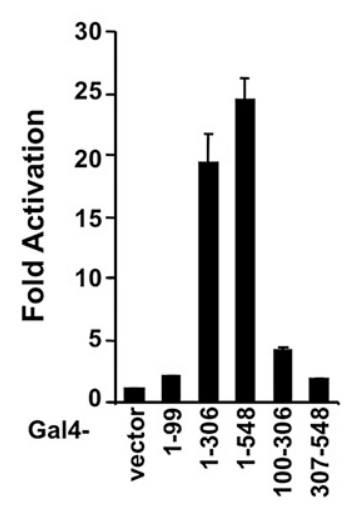

Figure 2. The novel HEB activation domain AD3 directly interacts with TFIID. (A) GST pull-down assays showing that the dTAFH domain of TAF4 interacts with E2A or HEB in Namalwa or HeLa nuclear extract, respectively. TFIIB and TFIIH served as controls. $(B)$ The eTAFH and dTAFH domains are differentially recognized by $\mathrm{HEB} \mathrm{AD} 1$ and $\mathrm{AD} 3$ domains, respectively. GST pull-down analyses of in vitro transcribed/translated HEB fragments (shown at left) binding to the GST-fused eTAFH or dTAFH domain. $(C, D)$. HEB AD3 directly interacts with TFIID. HeLa nuclear extract $(C)$ or the highly purified f-TBP-TFIID complex $(D)$ was subjected to GST pull-down assays with GST-fused HEB $\mathrm{AD} 1, \mathrm{AD} 2$, and $\mathrm{AD} 3 .(E) \mathrm{HEB} \mathrm{AD} 3$ is a novel transcriptional activation domain that acts synergistically with AD1. Luciferase reporter assays of the activities of Gal4-fused HEB fragments (as indicated in the left panel of $B$ ) on the pGL3$5 \times$ Gal4-E1B template; results were normalized to Renilla luciferase activities from a TK promoter and are presented relative to Gal4-DBD activity. Means \pm SD from triplicate experiments are shown. See also Supplemental Figure S2. previously unrecognized activation domain in similar reporter assays revealed (1) delocalization (based on additional $\mathrm{N}$-terminal and $\mathrm{C}$-terminal truncations) of the AD3 transcriptional activity to residues 221-300 (Supplemental Fig. S2A), (2) sequence conservation of the HEB AD3 domain in E-protein family members E2-2 (69\% identical) and E2A (32\% identical) (Supplemental Fig. S2B), (3) a corresponding conservation of the transcriptional function in E2A AD3 (Supplemental Fig. S2C), and (4) strong synergistic functions of AD3 with AD1 and/or AD2 (Supplemental Fig. S2D). Because the shorter HEB (residues 221-300) and corresponding E2A AD3 domains both showed higher activities than the broader domains (Supplemental Fig. S2C), the shorter AD3 domain was used in the following experiments.

The interaction of TFIID with HEB AD3 is dependent on the dTAFH domain of TAF4

The above results, showing that the TAF4 dTAFH domain interacts with HEB and E2A (Fig. 2A,B) and, reciprocally, that HEB AD3 directly binds TFIID (Fig. 2D), clearly suggested that the TAF4 dTAFH domain mediates the observed HEB/AD3-TFIID interactions. To test this hypothesis, we generated TFIID complexes containing either TAF4 wild type (TAF4wt) or TAFH-deleted TAF4 (TAF4A). To this end, HeLa lines that stably express Flagtagged TAF4wt or TAF4 $\Delta$ were established, and derived nuclear extracts were directly subjected to anti-Flag immunopurification. Consistent with a previous study /Guermah et al. 2001), mass spectrometric analyses revealed that TBP and all of the other normal TAF subunits were present in both f-TAF4-TFIID preparations (data not shown).
SDS-PAGE silver stain (Fig. 3A) and immunoblot (Fig. 3B) analyses also revealed comparable levels of TFIID subunits in both preparations. One potential complication with this approach is that human TFIID contains two copies of TAF4 (Guermah et al. 2001). However, since TAF4 $\Delta$ migrates faster than the endogenous (untagged) TAF4wt, it was possible to rule out any significant incorporation of endogenous TAF4 in these preparations (Fig. 3A,B). This exclusive incorporation of exogenous f-TAF4 proteins (both wt and $\Delta$ ) into TFIID complexes likely reflects their overexpression relative to the endogenous TAF4 but, in any case, simplifies the interpretation of subsequent experiments.

We next examined interactions of the purified f-TAF4TFIID complexes with an immobilized his-tagged Gal4AD3 fusion protein (h-Gal4-AD3) that contains the minimal AD3 domain. Consistent with the results of the binding assays with GST-HEB AD3 (amino acids 100306) (Fig. 2D) and the functional studies with Gal4-AD3 derivatives (Supplemental Fig. S1), the minimal HEB AD3 domain (residues 221-300) also interacts with the purified TFIID complex containing the TAF4wt subunit (Fig. 3C). Most importantly, however, HEB AD3 (221-300) shows no detectable interaction with the TAF4A TFIID complex lacking the TAF4 dTAFH domain (Fig. 3C). Therefore, we conclude that HEB AD3 interacts directly with the TFIID complex through the dTAFH domain of TAF4.

\section{The TFIID interaction is required for transcriptional activation by $H E B$ AD3}

The results described above showed that HEB AD3 exhibits a strong transcription activity in cells (Fig. 2E; 


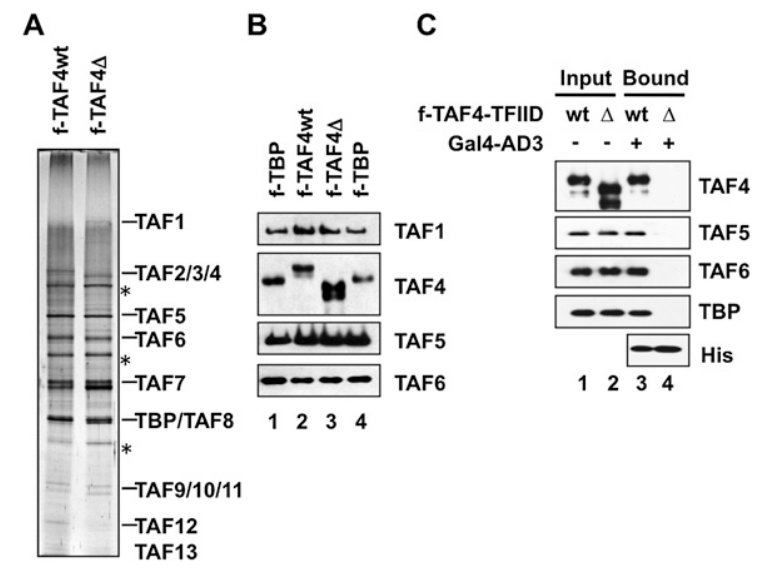

Figure 3. The TFIID interaction with HEB AD3 is dependent on the TAF4 dTAFH domain. $(A, B)$ Comparable levels of TFIID subunits are detected in purified f-TAF4 complexes. Silver staining $(A)$ and immunoblots of selected TAF subunits $(B)$ of M2 affinity-purified f-TAF4 complexes from HeLa cells expressing Flag-tagged wild-type (wt) or dTAFH-deleted $(\Delta)$ TAF4. The identities of the TFIID subunits were also confirmed by mass spectrometry (data not shown). The asterisks denote contaminants bound to $\mathrm{M} 2$ beads. $(C)$ Direct TFIID interaction with AD3 is dependent on the dTAFH domain of TAF4. Pull-down analyses of purified f-TAF4-TFIID complexes (as shown in $A$ ) by Ni bead-immobilized his-Gal4-HEB AD3 (221-300). Note that the minimal HEB AD3 fragment that shows activation was used here and in the following figures.

Supplemental Fig. S1C) and interacts directly with the TFIID complex through the TAF4 dTAFH domain (Fig. $3 \mathrm{C})$. To test the hypothesis that AD3 exerts its transcription function through its dTAFH-dependent interaction with the TFIID complex, we next established Gal4-AD3and ectopic TFIID-dependent in vitro transcription assays according to the protocol in Figure 4A. Consistent with the demonstrated activity of HEB AD3 in the luciferase reporter assays (Fig. 2D; Supplemental Fig. S2), the newly identified HEB AD3 domain showed strong transcriptional activity in the in vitro transcription assay (Fig. 4B). This activation is dependent on the TFIID interaction, since addition of an increasing amount of a dTAFH polypeptide gradually diminished the Gal4-AD3-mediated transcription but had no effect on transcription activation by Gal4-VP16 (Fig. 4B), which is thought to act through interactions with TAF6 and TAF12 (Liu et al. 2009). The latter result further attests to the overall structural integrity of the f-TAF4-TFIID complex. To more directly establish that the HEB AD3 activity is dependent on the TAF4 dTAFH domain, we carried out in vitro transcription assays using TFIID-depleted HeLa nuclear extract and supplementation with purified f-TAF4wt and f-TAF4A TFIID complexes (Fig. 3A). As expected, f-TAF4wt-TFIID supported both Gal4-AD3- and Gal4-VP16-dependent transcription (Fig. 4C, lanes 5,6). In contrast, f-TAF4 TFIID failed to support Gal4-AD3-mediated transcription (Fig. 4C, lane 5 vs. lane 6) but maintained full support of both Gal4-VP16-mediated and basal (activator-independent) transcription (Fig. 4C, lane 9 vs. lane 6, basal transcript from pML $\Delta 53$ ) as well as transcription by full-length p53 (Supplemental Fig. S3). These data indicate strongly that the dTAFH domain of the TAF4/ TFIID complex is selectively required for HEB AD3mediated transcription.

\section{HEB AD3 enhances TFIID binding to the core promoter dependent on the dTAFH domain of TAF4}

Whereas several studies have suggested roles for specific activator/activation domain-TAF interactions in TAFdependent transcription (see above), there is scant information on the underlying mechanisms. Among several possibilities, an enhanced recruitment of TFIID to the core promoter, a key rate-limiting step in gene activation, is an attractive and readily testable possibility. Therefore, to assess the mechanistic consequences of the HEB AD3-dTAFH/TFIID interaction, we carried out magnesium-agarose electrophoresis mobility shift (Mg-EMSA) (Lieberman and Berk 1994) and immobilized template (Black et al. 2006) assays using a DNA template containing five Gal4-binding sites upstream of the E1B

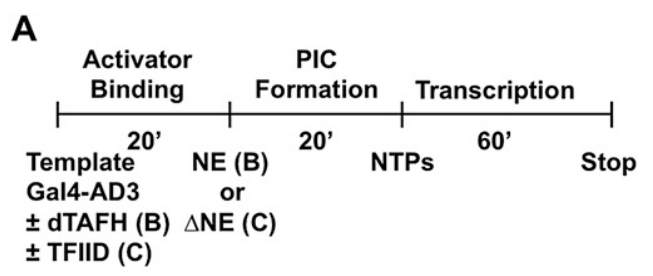

B

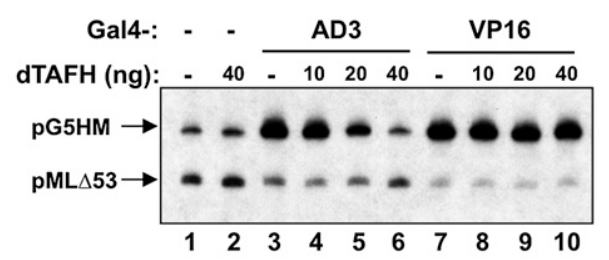

C

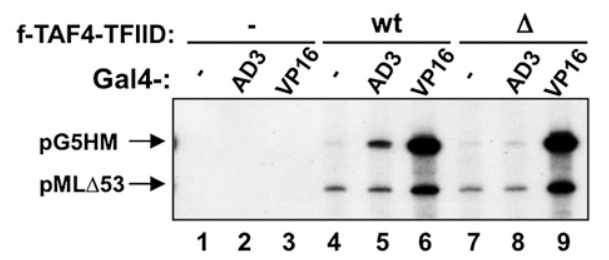

Figure 4. Transcriptional activation by HEB AD3 is dependent on the TAF4 dTAFH domain. (A) Schematic protocol for in vitro transcription assays. $(B)$ A dTAFH polypeptide selectively inhibits transcription activation by HEB AD3. Transcription from the Gal4-HM template by Gal4-HEB AD3 or Gal4-VP16 in the presence of increasing amounts of dTAFH polypeptide. $(C)$ The dTAFH domain of TAF4/TFIID is essential for HEB AD3mediated transcription activation. Transcription reactions were performed in TFIID-depleted HeLa nuclear extract $(\Delta \mathrm{NE})$ supplemented with equal amounts of f-TAF4-TFIID complexes containing either wild-type $(w t)$ or dTAFH-deleted $(\Delta)$ TAF4. Transcriptions from the Gal4-HM template with Gal4-HEB AD3 (221-300) or Gal4-VP16 and a control DNA template (pML $\Delta 53$ ) are indicated. See also Supplemental Figure S3. 
core promoter (Fig. 5A). In the EMSA assay, TAF4wt and TAF4 4 TFIID complexes showed equivalent levels of weak binding to the DNA probe in the absence of Gal4AD3. Importantly, the DNA binding of TAF4wt-TFIID was significantly enhanced by the addition of Gal4-AD3 (Fig. 5B, lane 5 vs. lane 3), whereas the binding of TAF4 4 -TFIID was only feebly enhanced (Fig. 5B, lane 4 vs. lane 6), clearly indicating that the Gal4-AD3enhanced binding is a direct result of the AD3-dTAFH interaction. Also of note, a parallel analysis with a template containing a mutated (TATA to GAGA) TATA box showed that the TATA box is absolutely required for basal and Gal4-AD3-enhanced TFIID binding (Fig. 5B, right panel), indicating the specificity of the TFIIDpromoter complexes and that Gal4-AD3 facilitates TFIID binding only at the core promoter. The enhancement of TFIID binding by Gal4-AD3 was also observed with a template containing only one Gal4-binding site (data not shown). To further confirm this result and show enhanced binding of an intact TFIID complex by Gal4-AD3, we also carried out immobilized template assays using the same DNA template. Consistent with the above results, we observed comparable levels of

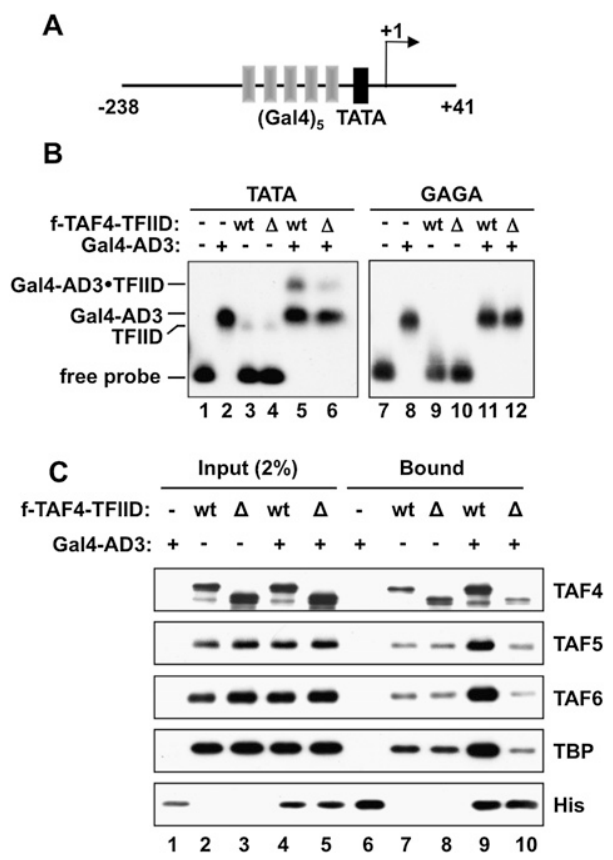

Figure 5. HEB AD3 enhances TFIID binding to DNA depending on the TATA box and the dTAFH domain of TAF4. $(A)$ Schematic representation of the DNA probe containing five Gal4 binding sites upstream of an E1B TATA box. (B) Mg-EMSA with DNA probes containing either wild-type (left) or mutated (right) TATA boxes, Gal4-AD3, and purified f-TAF4-TFIID complexes (TAF4wt or TAF4A) as indicated. Protein-DNA complexes were resolved by agarose gel electrophoresis and autoradiography. $(C)$ Immobilized template analyses of purified f-TAF4-TFIID complexes (TAF4wt or TAF4A) binding to the DNA probe in the presence or absence of Gal4-HEB AD3. DNAbound proteins were visualized by immunoblots of indicated TAF subunits, TBP, and His tag (for Gal4-HEB AD3). activator-independent binding of the TAF4 $\mathrm{wt}$ and TAF4 $\Delta$ TFIID complexes (monitored by TAF4, TAF5, TAF6, and TBP immunoblots) as well as a significant Gal4-AD3enhanced binding of TFIID that was selective for TAF4wt TFIID relative to TAF4 $\triangle$ TFIID (Fig. 5C). Overall, these results indicate (1) an HEB AD3-enhanced binding of TFIID that likely contributes to the AD3-enhanced transcription and (2) a significant role for the AD3-dTAFH interaction in the activator-enhanced TFIID binding to the core promoter.

HEB-mediated transcriptional activation of the natural p21 promoter is dependent on the TAFH-dependent coactivation function of TAF4

As an extension of the above-described studies that established a function and an underlying mechanism for the AD3-dTAFH/TAF4/TFIID interaction on a model promoter, it was also important to analyze this interaction in the more physiological context of full-length HEB-mediated activation of a natural promoter. For this objective, we used the human $p 21$ gene-proximal promoter that contains three E-box sites (Fig. 6A) and that was shown to be regulated by E2A in a reporter assay (Prabhu et al. 1997). Consistent with this observation, our studies of the endogenous $p 21$ gene in 293T cells revealed (1) that ectopic expression of E proteins (HEB, E12, E47, and E2-2) enhances both p21 mRNA and p21 protein levels (Supplemental Fig. S4A,B), (2) that depletion of HEB by shRNA treatment reduces the level of $p 21$ mRNA (Supplemental Fig. S4C,D), and (3) based on chromatin immunoprecipitation (ChIP) assays, that HEB binds near the $p 21$ transcription start site (TSS) and that HEB depletion results in decreased TFIID (monitored by TAF1) recruitment (Supplemental Fig. S4E).

Based on these results, we then selected $p 21$-proximal promoter and cognate activator HEB as a native model system for the following studies. In functional studies, we established a modified in vitro transcription assay (see the Materials and Methods for details) using a 5' linearized DNA template containing the $p 21$ promoter (nucleotide positions -133 to +42 ) upstream of a G-less cassette (Fig. 6B, top). Remarkably, purified recombinant HEB significantly enhanced transcription on the $p 21$ template in reactions with normal HeLa nuclear extract (Fig. 6B, bottom, lane 1 vs. lane 2). This result confirmed a direct function for HEB in activation of the $p 21$ gene. To further examine the predicted role of the dTAFH domain in TFIID-dependent transcription activation by HEB, we used TFIID-depleted nuclear extracts, which showed no basal or HEB-dependent activity (Fig. 6B, lanes 3,4 ), in conjunction with exogenous purified f-TAF4TFIID complexes. In these assays, TAF4wt-TFIID and TAF4 $\Delta$-TFIID restored equivalent levels of basal transcription from the $p M L \Delta 53$ template (Fig. 6B). Strikingly, however, HEB-dependent transcription was nearly fully restored by TAF4wt-TFIID but not by TAF4 $\Delta$-TFIID. These results clearly indicate the specificity and essential role of the dTAFH domain in HEB function on a natural promoter. 
A
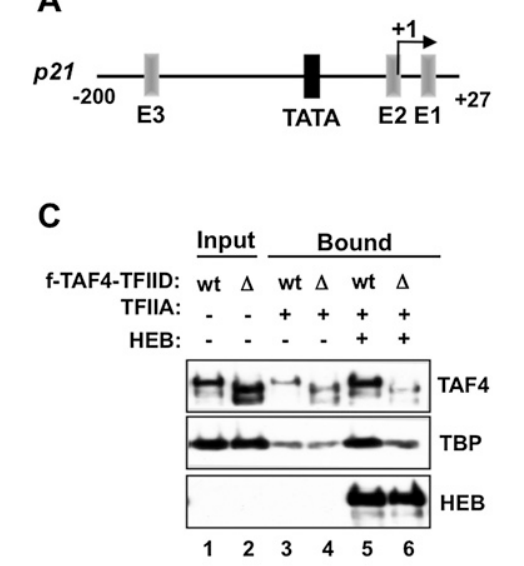

E

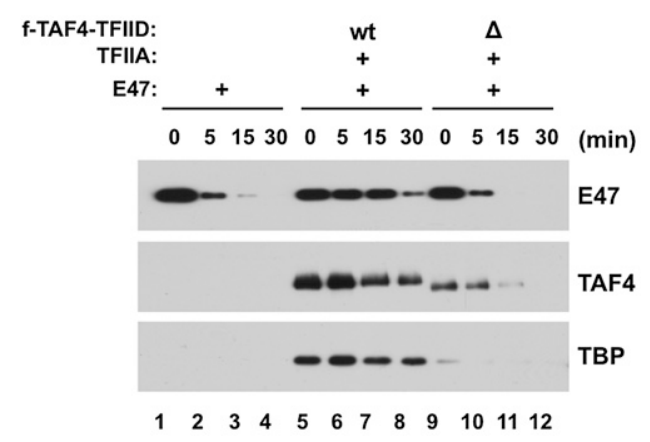

B

D
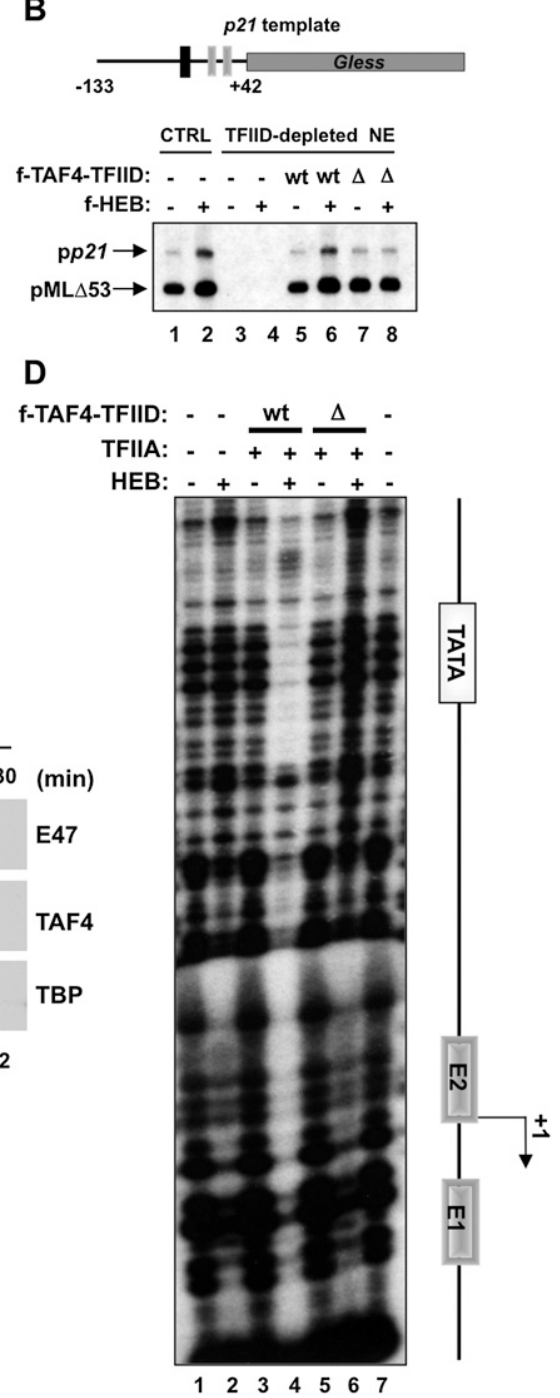

Figure 6. The dTAFH domain is important for HEB-mediated TFIID binding and transcription of the human $p 21$ promoter in vitro. (A) Schematic of the human $p 21$ core promoter region. $(B)$ In vitro transcription assays with the $p 21$ core promoter template (shown at the top), full-length HEB, and purified f-TAF4TFIID complexes (TAF4wt of TAF4A) as indicated in normal (lanes 1,2) or TFIID-depleted (lanes 3-8) HeLa nuclear extract. $(C, D)$ Immobilized template $(C)$ and DNase I footprinting $(D)$ assays showing that HEB-enhanced TFIID binding to the human $p 21$ core promoter is dependent on the dTAFH domain of TAF4. $(E)$ Immobilized template assay showing cooperative binding of TFIID and E47 on the $p 21$ core promoter. After an initial incubation at $4^{\circ} \mathrm{C}$ to allow binding, reactions were further incubated at $20^{\circ} \mathrm{C}$ to allow dissociation of DNAprotein complexes. Bead-immobilized templates were further washed at the indicated times, and bound proteins were monitored by immunoblot. See also Supplemental Figure S4.
HEB-mediated enhancement of TFIID binding to the natural p21 promoter is dependent on the TAFH domain of TAF4

To assess the mechanistic basis of the above-described effect of HEB on transcription of the $p 21$ promoter, we assessed the effect of HEB on TFIID binding by two independent methods. Since the $p 21$ promoter contains a weak TATA relative to that in the model promoter used in the analyses in Figure 5 and given many earlier studies indicating a stabilizing role of TFIIA on TFIID binding to such sites (Lieberman and Berk 1994), TFIIA was included in these analyses. In an initial immobilized templatebased assay, TAF4wt-TFIID and TAF4 $\Delta$-TFIID showed equivalent levels of basal (HEB-independent) binding (Fig. 6C, lanes 3,4). In contrast, HEB enhanced the binding of TAF4wt-TFIID but not TAF4 4 -TFIID (Fig. 6C, lane 8 vs. lane 6). These results are consistent with the selective effects of Gal4-AD3 on TAF4wt versus TAF4A TFIID binding and transcription.

To confirm and analyze in more detail the HEB- and dTAFH-dependent binding of TFIID to the $p 21$ promoter, we next carried out DNase I footprinting assays using the same DNA template. Consistent with the results of functional studies of the $p 21 \mathrm{E}$ boxes in luciferase assays (Prabhu et al. 1997), HEB bound directly to the two downstream E-box sites (Fig. 6D, lane 2 vs. lane 1) but not to the upstream E box (region not shown). Importantly, whereas neither TAF4wt-TFIID nor TAF4 $\Delta$-TFIID showed any DNA binding in the absence of HEB (Fig. 6D, lanes 3,5), HEB markedly enhanced the binding of TAF4wt-TFIID (evidenced by the strong protection over the TATA and downstream sequences), but not TAF4 $\Delta$ TFIID, to the promoter (Fig. 6D, lanes 3-6). Interestingly, a cooperative binding between HEB and TAF4wt-TFIID was also apparent, as evidenced by the enhanced protection over the E-box regions with HEB plus TAF4wtTFIID versus HEB alone (Fig. 6D, lane 4 vs. lane 2). To determine quantitatively the effect of this cooperative binding on E-protein binding, an immobilized template assay was performed using $\mathrm{E} 2 \mathrm{~A} / \mathrm{E} 47$, an $\mathrm{E}$ protein that binds more weakly to the $p 21$ promoter and rapidly dissociates from the $p 21$ template after incubation at 
$20^{\circ} \mathrm{C}$ (Fig. 6E). Notably, the temperature-dependent dissociation of E47 was significantly delayed by the addition of TAF4wt-TFIID (Fig. 6E, lanes 5-8) but not by TAF4 4 TFIID (Fig. 6E, lanes 9-12). As expected, the temperaturedependent loss of E47 binding in the presence TAF4 4 TFIID was accompanied by a corresponding loss of TFIID binding, whereas the delayed dissociation of E47 in the presence of TAF4wt-TFIID was accompanied by a reduced dissociation of TFIID (Fig. 6E). In summary, the above results firmly establish an E-protein-enhanced TFIID binding to the natural $p 21$ core promoter that involves a direct interaction of the E-protein $\mathrm{AD} 3$ domain with the TAF4 dTAFH domain and that correlates precisely with the dTAFH-dependent enhancement of transcription of $p 21$ by $\mathrm{E}$ proteins.

\section{The dTAFH domain of TAF4 is selectively required for TFIID recruitment and gene activation in vivo}

Having established a gene-selective TAF4 coactivator function and underlying mechanistic principles for HEBmediated activation of the $p 21$ promoter in vitro, we next examined the function of the TAF4 dTAFH domain in TFIID recruitment and function in a natural cellular context. These analyses used four cell lines based on the Taf $4^{\text {loxp/- }}$ and $\mathrm{Taf}^{-1-}$ fibroblast lines that were established from day 9.5 mouse embryos (Mengus et al. 2005). The $\mathrm{Taf}^{-1-}$ line was used to establish stable lines that express either Flag-tagged human TAF4wt

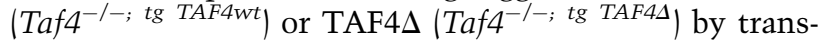
fection with corresponding TAF4 expression vectors. For our analyses, we selected cell lines that were shown by immunoblot to express ectopic human TAF4 proteins at levels comparable with that of the endogenous murine TAF4 (Fig. 7A). Importantly, reciprocal immunoprecipitation assays (with anti-TAF1 and anti-TAF4) showed efficient and comparable incorporation of exogenous f-TAF4wt and f-TAF4 $\Delta$ into the murine TFIID complexes (Fig. 7A).

To identify TAFH/TAF4-dependent gene activation, genome-wide RNA sequencing (RNA-seq) analyses were executed in these stable MEF lines and confirmed the deletion of exons 11 and 12 of the Taf4 gene in the Taf4 ${ }^{-/-}$ MEF (Mengus et al. 2005) and its derived lines (Supplemental Fig. S5). Since clonal selection could result in variation of gene expression in cultured cells, we focused on the differentially expressed genes that were commonly dependent on endogenous or exogenous TAF4. We found 401 genes with a greater than twofold up-regulation and 81 genes with a greater than twofold down-regulation that were dependent on endogenous or exogenous wildtype TAF4 but only 10 up-regulated and 15 down-regulated genes whose TAF4 dependence was restored by TAF4A (Fig. 7B). Correlation clustering of all differentially expressed genes also suggests a high degree of correlation between f-TAFwt-expressing cells and $\mathrm{Taf} 4^{\text {lox/- }}$ cells, whereas f-TAF4 $\Delta$-expressing cells are similar to $\mathrm{Taf}^{-/-}$ cells (Supplemental Fig. S6). It is noteworthy that the RNA-seq analysis also showed a requirement of TAFH/ TAF4 for p21 gene activation (Fig. 7C). However, among
TAF4-regulated genes, we could not identify any significant cellular pathways by gene ontology analysis or any consensus motifs for transcription factor binding by analysis of corresponding promoter regions (up $-1 \mathrm{~kb}$ relative to the TSS). These results are consistent with those of an earlier study of TAF4 function by microarray profiling (Mengus et al. 2005). Collectively, these results strongly suggest that the TAFH domain is globally required for TAF4 function in vivo and that TAFH functions may involve interactions with diverse activators.

To assess TFIID recruitment to the TSS of $p 21$, ChIP assays were performed using an anti-TAF1 antibody. As shown in Figure 7D for selected genes (from genome-wide profiling), including $p 21$, TFIID binding (monitored with anti-TAF1 antibody) was detected in cell lines expressing endogenous murine TAF4 (blue bars) or exogenous human TAF4wt (green bars) but not in the TAF4-null line (red bars) or the cell line expressing TAF4D (purple bars). Consistent with the concept of TFIID as an essential transcription initiation factor, the binding of Pol II tightly correlated with the binding of TFIID to the p21 TSS /data not shown). These results and the biochemical analyses in Figure 6 clearly indicate that the dTAFH domain of TAF4 is required for TFIID recruitment to the $p 21$ core promoter. Interestingly, this dTAFH-dependent TFIID recruitment was also observed on other genes, including Gata 6, Dcn, Pdgfr-a, and Serpinf1. In contrast, TFIID recruitment to two of the examined genes, Itga11 and Mrpplf3, appeared TAF4-dependent but dTAFH-independent. Moreover, among the examined genes, we also found two, Fth1 and Mif, for which TFIID recruitment was TAF4-independent. Notably, for the genes analyzed, the relative mRNA levels in the different cell lines showed striking parallels to the levels of TFIID recruitment, further confirming the function of dTAFH-dependent TFIID recruitment in gene activation (Fig. 7E). Collectively, these observations (1) confirm the physiological relevance of the dTAFH-dependent physical and functional interactions of TAF4/TFIID observed in the biochemical analyses, (2) establish gene-selective TAF4and dTAFH-dependent functions, and, related, (3) raise the possibility that dTAFH-dependent interactions may have important roles in gene activation not only by E proteins, but also by other activators.

\section{Discussion}

The activator-dependent recruitment of diverse transcription coactivators to gene promoters is a central theme in transcription activation. In the strict sense of coactivator function emphasized here, this recruitment is mediated by direct physical contacts between activators and coactivators. Various biochemical and genetic analyses (see above) have shown that the TAFs within initiation factor TFIID are required for activator-dependent transcription and, in particular, are apparent direct targets of an increasing number of activators. However, interactions in the context of the TFIID complex and corresponding functional relevance on natural promoters have not been established in most cases, and even for the 
Chen et al.

A

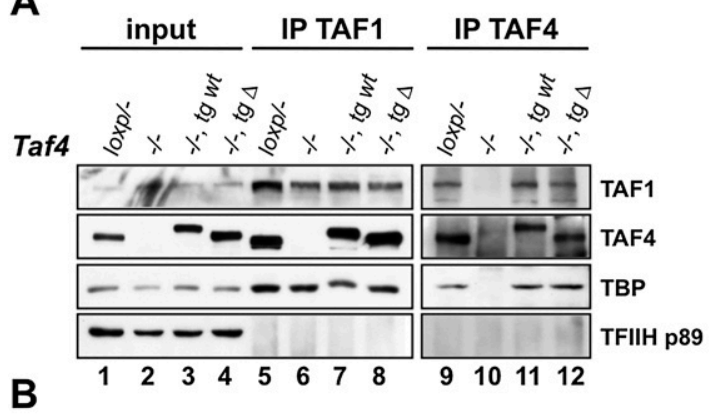

C

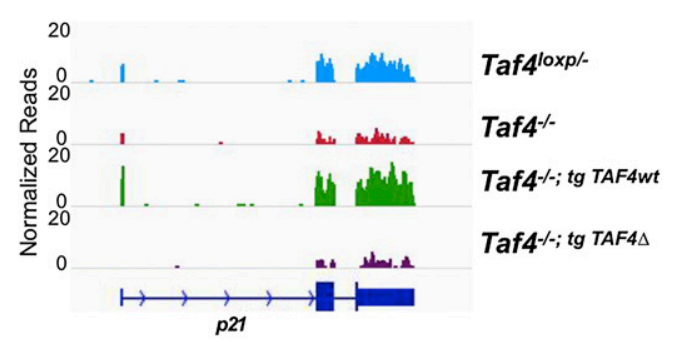

\section{Up-regulated $\geq 2$ fold}

(vs Taf $4 \%, q<0.05$ )

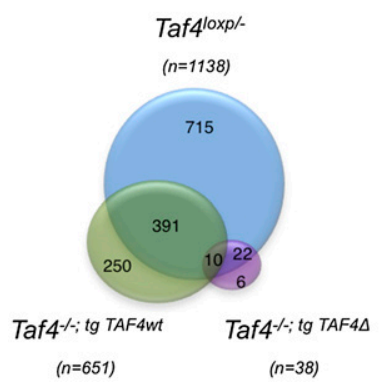

D
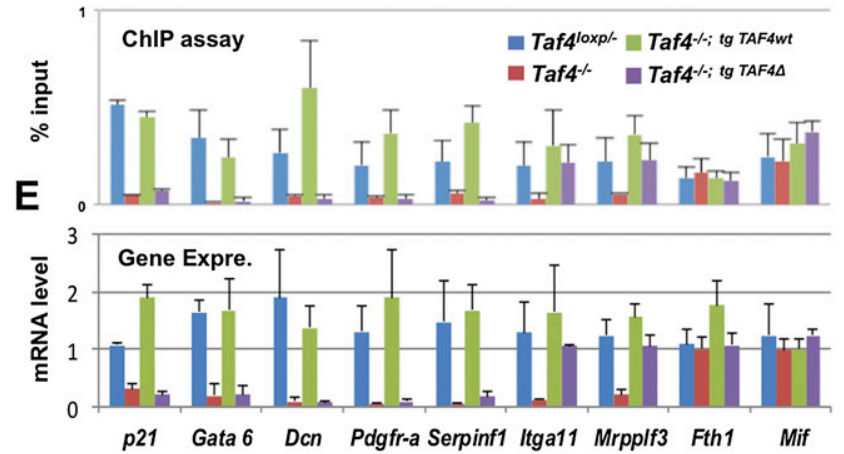

Down-regulated $\leq 0.5$ fold

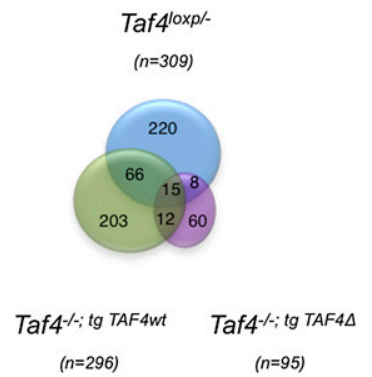

(vs Taf4 $\%, \mathrm{q}<0.05$ )

\section{$\mathbf{F}$}
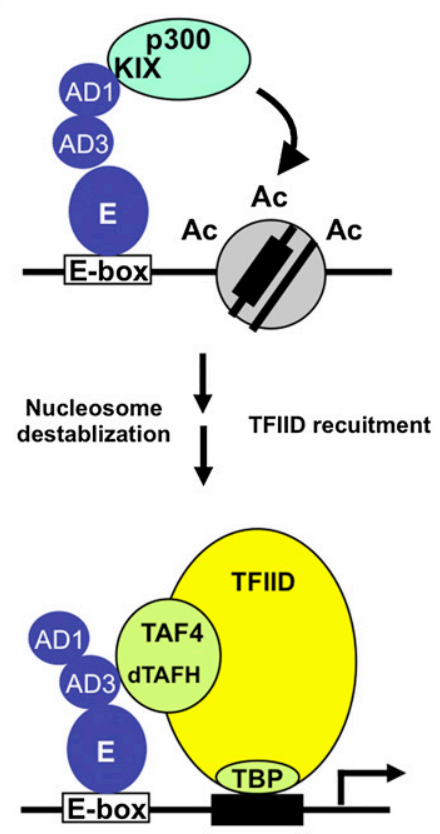

Figure 7. The dTAFH domain of TAF4 is crucial for TFIID recruitment and transcriptional activation in vivo. $(A)$ Coimmunoprecipitation assays showing efficient incorporation of human TAF4 into the endogenous murine TFIID complex. Cell lysates of stable MEF lines were subjected to immunoprecipitation with anti-TAF1 or anti-TAF4 antibodies. Immunoblots of immune complexes with the indicated antibodies are shown. TFIIH p89 is a control. $(\mathrm{tg})$ Transgene. $(B)$ Venn diagrams showing the numbers of genes differentially expressed with greater than twofold up-regulation (left) or down-regulation (right) in the indicated cell lines relative to $\mathrm{Taf4}^{-1-}$ MEFs. Circle size is proportional to the number of genes. $(C)$ Representative RNA-seq gene track at the $p 21$ locus in stable MEF lines. $(D)$ ChIP analyses of TAF1 (representative of TFIID) recruitment at the TSSs of the indicated genes in stable MEF lines. $(E)$ Expression levels of the indicated genes in stable MEF lines. Means \pm SD from triplicate experiments are shown. $(F)$ Working model for E-protein activation domain-dependent recruitment of coactivators in transcriptional activation (see the Discussion for details). Note that while the universal Mediator is likely involved in E-protein target gene activation as well, the model emphasizes only those mammalian coactivators for which E-protein interactions are well documented. See also Supplemental Figures S5 and S6.

best documented cases of relevant activator-TFIID interactions, mechanistic details-in particular, activator effects on TFIID recruitment versus other aspects of TFIID function-on natural target promoters have been lacking.

Through a combination of rigorous biochemical and cellular approaches, we show here (1) the presence of a new and highly potent activation domain (AD3) within E proteins; (2) a specific and direct interaction between
HEB and TAF4 within the context of TFIID through an HEB AD3-TAF4 dTAFH domain interaction; (3) that the AD3-dTAFH interaction is essential for HEB- and HEB AD3-mediated transcription activation; (4) mechanistically, that the AD3-dTAFH interaction contributes critically to AD3/HEB-enhanced TFIID binding to a natural promoter; (5) that the dTAFH domain also plays an important gene-selective role in TFIID recruitment and TAF4/TFIID-mediated transcription activation in vivo; and 
(6) that TAF4 function on the large genomic group ( 400) of TAF4-dependent genes depends almost uniformly on the TAFH domain, implying a common mechanism for TAF4 function. These results provide definitive evidence for the long-standing but poorly documented hypothesis that TAFs within the TFIID complex can function as bona fide gene-selective coactivators through direct interactions with promoter-bound activators on natural genes. They also establish a clear mechanism of action for TAF4 coactivator function involving activator-dependent recruitment of TFIID to cognate natural promoters. They are also of major significance in relation to the key role of $\mathrm{E}$ proteins in development-notably in lymphopoiesis (Quong et al. 2002; Kee 2009) and leukemogenesis (Aspland et al. 2001; Seidel and Look 2001).

\section{Activator- and gene-selective TAF4 coactivator functions through the dTAFH domain and associated activator-TFIID interactions}

Our identification of the potent AD3 activation domain (discussed below) in HEB and E2A and a corresponding direct interaction domain (dTAFH) in the TAF4 subunit of TFIID allowed a critical test of the idea (see above) that direct activator-TAF/TFIID interactions are involved in TAF coactivator functions. In support of this hypothesis, our biochemical analyses demonstrated (1) a direct interaction of $\mathrm{HEB}$ or the isolated $\mathrm{AD} 3$ with TFIID as well as a direct interaction of HEB with TAF4 in the context of TFIID, (2) an impaired interaction of HEB/AD3 with a reconstituted TFIID complex lacking the dTAFH domain in TAF4, and (3) a corresponding loss of transcriptional activation by HEB on the natural p21 promoter or by Gal4-fused AD3 on a model promoter with the reconstituted TFIID complex lacking the dTAFH domain in TAF4. Importantly, TFIID-dependent transcriptional activation by p53 and Gal4VP16 was unaffected by deletion of the dTAFH domain in TAF4. These in vitro results established a direct TAF4 coactivator function (involving dTAFH-AD3 interactions) for transcriptional activation by HEB or AD3 as well as activator-selective functions of the dTAFH domain. In confirmation of these results, complementation assays with $\mathrm{Taf4}^{-/-}$fibroblasts revealed restoration of transcription of the HEB/E2A-activated TAF4-dependent $p 21$ gene by intact TAF4 but not by TAFH-deleted TAF4.

Of note, our genome-wide gene expression profiles established that $97 \%$ (391 out of 401) of the TAF4dependent genes that were commonly activated by endogenous or exogenous wild-type TAF4 were also dTAFH domain-dependent. These results strongly suggest that the TAFH domain plays a crucial role in a broad range of activator-TAF4/TFIID interactions. In support of this notion, a recent phage display screen for dTAFH-interacting peptides identified sequences resident in several known activators (Wang et al. 2007), and a study in Drosophila revealed that a broad region of TAF4 containing the dTAFH domain interacts with Pygopus and is required by an unknown mechanism for Wingless-induced activation of the $n k d$ gene by Pygopus (Wright and Tjian 2009).

In relation to broader functions of TAF4 that may involve other activation domains, it is notable that TAF4 is the TFIID-associated TAF for which the largest number of interacting activators has been identified (see above). Furthermore, our complementation analyses of $\mathrm{Taf4}^{-1-}$ fibroblasts also revealed that dTAFHdeleted TAF4, like TAF4, is able to fully rescue expression of 10 TAF4-dependent genes, clearly indicating gene-selective functions for the TAFH domain. This result is consistent with the idea, supported by studies of Sp1 and CREB (Hoey et al. 1990; Saluja et al. 1998), that TAF4 contains other (e.g., glutamine-rich) domains that can be used by distinct regulatory factors. Consistent with our genome-wide gene expression profiles, an earlier microarray profiling showed that Taf4 deletion in MEFs results in deregulated expression of at least 1000 genes (Mengus et al. 2005). These results also suggest a broad range of TAF4 functions, although many of the deregulated genes could be indirect targets of TAF4.

\section{Mechanisms underlying transcriptional activation by the HEB/AD3-dTAFH/TAF4 interaction}

Core promoter-bound TFIID plays a key role in initiating assembly of the PIC, such that recruitment and stabilization of TFIID represents a key regulatory step. Mechanisms for activator-independent binding and/or stabilization of TFIID at the core promoter include (1) recognition by TBP and TAFs of core promoter elements (JuvenGershon et al. 2008), (2) enhancement of TFIID binding by TFIIA (Thomas and Chiang 2006), and (3) recognition of trimethylated H3K4 by TAF3 and acetylated H3 and H4 by TAF1 (Thomas and Chiang 2006; Goodrich and Tjian 2010). Direct activator interactions with TFIID offer a complementary means for TFIID recruitment and stabilization. Indeed, very early studies showed that activators can have both quantitative (enhanced recruitment) and qualitative (extended downstream DNA interactions) effects on TFIID binding (Sawadogo and Roeder 1985; Abmayr et al. 1988; Horikoshi et al. 1988). Related early studies showed activator-enhanced formation of a rate-limiting TFIID-TFIIA-promoter complex involving extended downstream interactions (isomerization) of TFIID on model promoters with multimerized activator-binding sites (Lieberman and Berk 1994; Chi and Carey 1996), although the effects may have been indirect through activator-TFIIA interactions (Kobayashi et al. 1995). Along with later demonstrations of catalytic activities in TAF1, these results suggested that activatorTFIID interactions may act mechanistically to (1) enhance TFIID recruitment, (2) alter conformational/promoter binding states of TFIID (isomerization) that affect downstream steps in PIC formation/function (Roeder 1996), and (3) regulate the enzymatic functions of TAF1/ TFIID (Thomas and Chiang 2006).

Here, our biochemical analyses demonstrated a clear ability of HEB to enhance the level of TFIID binding to 
the natural $p 21$ promoter. Although this enhanced TFIID binding requires the presence of TFIIA because of the weak p21 TATA element, it nonetheless must reflect a direct HEB-TFIID interaction because of the dependence on the AD3-interacting TAF4 dTAFH domain. This idea is confirmed by our demonstration that AD3 enhances TFIID binding to the TATA-containing E1B promoter in the absence of TFIIA but is dependent on the TAF4 dTAFH domain. Thus, given the direct interactions of HEB/AD3 with TFIID through the TAF4 dTAFH domain and the dTAFH-dependent transcriptional activation by $\mathrm{HEB} / \mathrm{AD} 3$ (discussed above), these results clearly indicate that $\mathrm{HEB} / \mathrm{AD} 3$-mediated transcriptional activation involves an $\mathrm{HEB} / \mathrm{AD} 3$-mediated enhancement of TFIID recruitment/promoter binding. In strong support of the physiological relevance of these observations, our ChIP analyses in the $\mathrm{Taf}^{-/-}$MEF complementation assays showed a clear dTAFH/TAF4-dependent recruitment of TFIID to endogenous dTAFH/TAF4-dependent genes (including $p 21$ ).

Whereas our data clearly indicate an effect of HEB on transcription of the natural $p 21$ gene through direct HEBTAF4/TFIID interactions that enhance TFIID binding, the biochemical (DNase footprinting and immobilized template) analyses also indicated a reciprocal effect of TFIID on HEB/E47 binding. This result, which parallels our earlier demonstration of highly cooperative binding of USF and TFIID to the adenovirus major late promoter (Sawadogo and Roeder 1985), suggests additional coactivator functions for activator-interacting TAFs, as the stabilized activator binding may facilitate activator interactions with other cofactors (Naar et al. 2001; Roeder 2005 ) in the overall activation pathway.

\section{Differential functions of the homologous TAF4 dTAFH and ETO eTAFH domains}

Our previous biochemical and structural analyses revealed that the eTAFH domain strongly and directly binds an LxxLL-containing motif of the AD1 domain of E proteins (Zhang et al. 2004; Plevin et al. 2006). In this study, surprisingly, we found that the homologous dTAFH domain preferentially interacts with the AD3 domain and not, as anticipated, with the AD1 domain. This result is consistent with the apparent absence of an LxxLL-like motif in the AD3 domain (Supplemental Fig. S2B). These results suggest that, despite their high degree of similarity, eTAFH and dTAFH domains may act mainly through distinct binding partners. In agreement with these observations, the two TAFH domains exhibit structural differences that likely affect their binding specificity (Plevin et al. 2006; Wang et al. 2007). Notably, in relation to alternative HEB interactions, the AD1-eTAFH interaction facilitates repression of HEB target genes by both blocking AD1-p300 interactions (below) and effecting corepressor ETO (and associated HDAC) recruitment to HEB target genes (Zhang et al. 2004), while the AD3-dTAFH interaction facilitates activation of HEB target genes by TAF4/TFIID recruitment.
Multiple E-protein activation domains: functional cooperativity and implications for biological functions of $E$ proteins

Although there has been substantial progress in understanding the biological context and pathways (including specific target genes) in which E proteins function (Quong et al. 2002; Kee 2009), mechanisms underlying E-protein functions have been poorly understood. Previous studies have revealed two conserved activation domains, AD1 and $\mathrm{AD} 2$, with important roles in E-protein-mediated transcription. Mechanistically, a direct interaction between AD1 (through an N-terminal $\alpha$ helix) and the histone acetyltransferase p300/CBP (through the KIX motif) has been shown (Bayly et al. 2004), whereas AD2 has been reported to interact (albeit only in yeast) with the SAGA histone acetyltransferase complex (Massari et al. 1999). In this study, we identified a novel activation domain that, as discussed above, exhibits a robust transcription function both in vivo and in vitro and appears to contribute to E-protein function primarily through interactions with TFIID. Although E2A AD3 shows only modest homology with HEB AD3, it also showed (as a Gal4 fusion protein) a robust transcription activity in a reporter assay. These results indicate that $\mathrm{AD} 3$, like $\mathrm{AD} 1$ and $\mathrm{AD} 2$, is also highly conserved in E proteins and also are consistent with the demonstration of partially redundant E-protein functions (Kee 2009).

Notably, and consistent with apparent differences in their primary mechanisms of action, reporter assays with Gal4-HEB fusion proteins demonstrated that AD3 can act synergistically with both $\mathrm{AD} 1$ and $\mathrm{AD} 2$, whereas the joint deletion of all three activation domains completely abrogates the transcription activity of Gal4-HEB. This result is consistent with a recent study (Bhalla et al. 2008) showing that an E2A mutant lacking both AD1 and AD2 retains the ability to promote $\mathrm{B}$ lymphopoeisis with associated activation of B-lineage genes when ectopically expressed in primary $E 2 A^{-/-}$fetal liver multipotent progenitors. This likely reflects the activity of the residual AD3 domain in the ectopic E2A, since expression of the bHLH (DNA-binding) domain of E2A failed to support B-cell development and gene activation. However, as histone acetyltransferase recruitment by $\mathrm{E}$ proteins is essential for activation of endogenous B-lineage genes, the AD1/AD2-deficient E2A might use other domains, including $\mathrm{AD} 3$, to directly or indirectly recruit an acetyltransferase. Consistent with the residual activity of the overexpressed AD1/AD2-deficient E2A in B-cell precursors, the HEB AD3 domain (as a Gal4 fusion) is also capable of activating a chromosomally integrated luciferase reporter gene containing multiple Gal4-binding sites in 293T cells (data not shown). Apart from the possibility of a direct interaction of the AD3 domain with an acetyltransferase, the AD3-recruited TFIID complex might also contribute a histone acetyltransferase activity through TAF1 (Mizzen et al. 1996). Collectively, these results suggest that AD3 is functionally conserved and plays an important role in the transcription regulation by $\mathrm{E}$ proteins. 
Model for regulation of E-protein-mediated transcription by interacting cofactors

Based on previous and current results, we present a working model for the regulation of E-protein function by their divergent activation domains and interacting coactivators (Fig. 7F). In this model, enhancer-bound E proteins recruit the coactivators $\mathrm{p} 300 / \mathrm{CBP}$, the major histone acetyltransferases implicated in E-protein function, through a direct interaction between the E-protein $\mathrm{AD} 1$ domain and the p300/CBP KIX domain (Bayly et al. 2004). Subsequent p300/CBP-mediated acetylation of core histones leads to destabilization of nucleosomes around the core promoter region, with increased accessibility to core promoter recognition factors such as TFIID and TFIIA. The direct interaction of E-protein AD3 with the dTAFH domain of TAF4, possibly after autoacetylation-mediated dissociation of p300 (Black et al. 2006), facilitates TFIID recruitment and stabilization in conjunction with TFIID interactions with core promoter elements. Finally, the reciprocal TFIID-mediated stabilization of E-protein binding may facilitate E-protein interactions with other coactivators.

\section{Materials and methods}

Antibodies, plasmids, and recombinant proteins

Details regarding the antibodies, plasmids, and recombinant proteins used in this study are provided in the Supplemental Material.

\section{Cell lines}

Taf4 ${ }^{\text {loxp/- }}$ and Taf4 ${ }^{-/-}$MEF cell lines (Mengus et al. 2005) were generously provided by Drs. Gabrielle Mengus and Irwin Davidson (Institut de Génétique et de Biologie Moléculaire et Cellulaire, Strasbourg). Stable lines were established by transfecting HeLa S3 or Taf4 ${ }^{-/-}$MEF cells with pIRESneo vectors encoding either f-TAF4wt or dTAFH $\Delta$ forms as indicated. Clonal cells were selected by treatment with $500 \mu \mathrm{g} / \mathrm{mL}$ G418 for $2 \mathrm{wk}$ and screened by immunoblotting with anti-Flag M2 and anti-TAF4 antibodies for positive clones. HeLa S3 and MEF cell lines were maintained in DMEM supplemented with 10\% FBS.

\section{Purification of TFIID complexes}

The preparation of HeLa nuclear extracts and purification of the f-TBP-TFIID complex from HeLa 3-10 cells were described previously (Chiang et al. 1993). For purification of f-TAF4-TFIID complexes, nuclear extracts from f-TAF4 HeLa S3 lines were incubated with anti-Flag M2 agarose in BC300, 0.1\% NP40 for $4 \mathrm{~h}$ at $4{ }^{\circ} \mathrm{C}$ and extensively washed with BC300 and $0.1 \%$ NP40. The bound protein complexes were eluted in $\mathrm{BC} 100$ and $0.1 \%$ NP40 supplemented with $0.15 \mathrm{mg} / \mathrm{mL} 3 x$ Flag peptide (Sigma). The TBP levels of eluted f-TAF4 complexes were determined by TBP immunoblotting along with recombinant TBP as standards. The concentrations of TFIID complexes were adjusted to $5 \mathrm{ng} / \mu \mathrm{L}$ TBP.

\section{In vitro transcription assays}

The preparation of TFIID-depleted nuclear extracts and transcription reactions for Gal4-HEB AD3 (221-300) and Gal4-VP16 were performed essentially as described (Oelgeschlager et al.
1998) with some modifications. Briefly, the $p G 5 H M C 2 A T$ template was used for activator-dependent transcription, whereas the $p M L \Delta 53 C 2 A T$ was used for activator-independent transcription. Reactions were supplemented with f-TAF4-TFIID complexes containing $25 \mathrm{ng}$ of TBP as indicated. The pp21C2AT template was constructed by cloning a fragment $(-133$ to +42$)$ of the human $p 21$ promoter into the EcoRI/EagI sites of the $p C 2 A T$ vector. For transcription reactions with HEB, EcoRI-digested DNA templates were gel-purified and incubated with $100 \mathrm{ng}$ of f-HEB and f-TAF4-TFIID complexes (25 ng of TBP) before addition of nuclear extracts and NTPs (without 3'-O-Me-GTP). RNA transcripts were digested with RNase $\mathrm{T} 1$, purified, and analyzed by electrophoresis and autoradiography.

Mg-EMSA, immobilized template protein recruitment (ITA), and DNase I footprinting assays

The Mg-EMSA (Lieberman and Berk 1994), ITA (Black et al. 2006), and DNase I footprinting assays (Guermah et al. 2001) were performed as previously described. Details can be found in the Supplemental Material.

\section{Pull-down, coimmunoprecipitation, and reporter assays}

Standard procedures were used and details can be found in the Supplemental Material.

\section{ChIP/RT-qPCR assays}

Standard procedures for ChIP-qPCR and RT-qPCR were used. ChIP results are shown as percentage of input DNA. Gene expression levels were normalized against Hprt. Details and primer sequences can be found in the Supplemental Material.

\section{Genome-wide RNA-seq analysis}

Total RNA from MEF lines (for Taf4 ${ }^{\text {loxp/- }}$ and $\mathrm{Taf4}^{-/-}$, two biological replicates in each line; for f-TAF4wt and f-TAF4 $\Delta$, two independent clones in each line) were extracted and subjected to high-throughput sequencing. Illumina multiplexing library construction, HiSeq 2000 SR50 sequencing, and raw data generation were performed by the Epigenomics Core Facility at Weill Cornell Medical College (New York, NY) according to the manufacturer's instructions. Raw image data were converted into base calls and fastq files via the Illumina pipeline CASAVA version 1.8 with default parameters. All 50-base-pair-long reads were mapped to the reference mouse genome sequence $\mathrm{mm} 9$ using TopHat with the default parameters (Trapnell et al. 2009). The mRNA level for each expressed gene/transcript was represented as FPKM (fragments per kilobase of transcript per million fragments mapped), and differential expression levels between cell lines were analyzed by Cuffdiff in Cufflinks software (Trapnell et al. 2010). For a statistically significant difference, a $Q$-value (false discovery rate) of $<0.05$ and a greater than twofold change were used.

\section{Data access}

RNA-seq data (fastq, bigwig, and Excel tables for gene expression profiles) have been deposited into the Gene Expression Omnibus (GEO) database (accession no. GSE46807).

\section{Acknowledgments}

We are grateful to Drs. Gabrielle Mengus and Irwin Davidson (Institut de Génétique et de Biologie Moléculaire et Cellulaire, 
Strasbourg) for Taf4 ${ }^{\text {loxp/- }}$ and Taf4 ${ }^{-/-}$MEF cells, and members of the Roeder laboratory for helpful discussions. We also thank Sohail Malik for useful comments on the manuscript, and Yushan Li (Weill Cornell Medical College, New York) for technical support. J.Z. was a Leukemia and Lymphoma Society Special Fellow (3286-04). W.-Y.C was a Leukemia and Lymphoma Society Fellow (5230-09) and a recipient of a Taiwan Merit Scholarship (NSC-095-SAF-I-564-603-TMS). This work was supported by grants from the NIH (CA163086, CA129325, and CA113872) and the Starr Cancer Consortium to R.G.R.

\section{References}

Abmayr SM, Workman JL, Roeder RG. 1988. The pseudorabies immediate early protein stimulates in vitro transcription by facilitating TFIID: Promoter interactions. Genes Dev 2: 542553.

Albright SR, Tjian R. 2000. TAFs revisited: More data reveal new twists and confirm old ideas. Gene 242: 1-13.

Aspland SE, Bendall HH, Murre C. 2001. The role of E2A-PBX1 in leukemogenesis. Oncogene 20: 5708-5717.

Bayly R, Chuen L, Currie RA, Hyndman BD, Casselman R, Blobel GA, LeBrun DP. 2004. E2A-PBX1 interacts directly with the KIX domain of $\mathrm{CBP} / \mathrm{p} 300$ in the induction of proliferation in primary hematopoietic cells. I Biol Chem 279: 55362-55371.

Bhalla S, Spaulding C, Brumbaugh RL, Zagort DE, Massari ME, Murre C, Kee BL. 2008. differential roles for the E2A activation domains in $\mathrm{B}$ lymphocytes and macrophages. I Immunol 180: 1694-1703.

Black JC, Choi JE, Lombardo SR, Carey M. 2006. A mechanism for coordinating chromatin modification and preinitiation complex assembly. Mol Cell 23: 809-818.

Burley SK, Roeder RG. 1996. Biochemistry and structural biology of transcription factor IID (TFIID). Annu Rev Biochem 65: 769-799.

Chatterjee S, Struhl K. 1995. Connecting a promoter-bound protein to TBP bypasses the need for a transcriptional activation domain. Nature 374: 820-822.

Chi T, Carey M. 1996. Assembly of the isomerized TFIIATFIID-TATA ternary complex is necessary and sufficient for gene activation. Genes Dev 10: 2540-2550.

Chiang CM, Ge H, Wang Z, Hoffmann A, Roeder RG. 1993. Unique TATA-binding protein-containing complexes and cofactors involved in transcription by RNA polymerases II and III. EMBO J 12: 2749-2762.

Garbett KA, Tripathi MK, Cencki B, Layer JH, Weil PA. 2007. Yeast TFIID serves as a coactivator for Raplp by direct protein-protein interaction. Mol Cell Biol 27: 297-311.

Goodrich JA, Tjian R. 2010. Unexpected roles for core promoter recognition factors in cell-type-specific transcription and gene regulation. Nat Rev Genet 11: 549-558.

Green MR. 2000. TBP-associated factors (TAFIIs): Multiple, selective transcriptional mediators in common complexes. Trends Biochem Sci 25: 59-63.

Guermah M, Tao Y, Roeder RG. 2001. Positive and negative TAF(II) functions that suggest a dynamic TFIID structure and elicit synergy with traps in activator-induced transcription. Mol Cell Biol 21: 6882-6894.

Hoey T, Dynlacht BD, Peterson MG, Pugh BF, Tjian R. 1990. Isolation and characterization of the Drosophila gene encoding the TATA box binding protein, TFIID. Cell 61: 11791186.

Hoffmann A, Sinn E, Yamamoto T, Wang J, Roy A, Horikoshi M, Roeder RG. 1990. Highly conserved core domain and unique
$\mathrm{N}$ terminus with presumptive regulatory motifs in a human TATA factor (TFIID). Nature 346: 387-390.

Horikoshi M, Hai T, Lin YS, Green MR, Roeder RG. 1988. Transcription factor ATF interacts with the TATA factor to facilitate establishment of a preinitiation complex. Cell 54: 1033-1042.

Juven-Gershon T, Hsu JY, Theisen JW, Kadonaga JT. 2008. The RNA polymerase II core promoter-the gateway to transcription. Curr Opin Cell Biol 20: 253-259.

Kee BL. 2009. E and ID proteins branch out. Nat Rev Immunol 9: $175-184$.

Kobayashi N, Boyer TG, Berk AJ. 1995. A class of activation domains interacts directly with TFIIA and stimulates TFIIATFIID-promoter complex assembly. Mol Cell Biol 15: 64656473.

Layer JH, Miller SG, Weil PA. 2010. Direct transactivatortranscription factor IID (TFIID) contacts drive yeast ribosomal protein gene transcription. I Biol Chem 285: 1548915499.

Li B, Carey M, Workman JL. 2007. The role of chromatin during transcription. Cell 128: 707-719.

Lieberman PM, Berk AJ. 1994. A mechanism for TAFs in transcriptional activation: Activation domain enhancement of TFIID-TFIIA-promoter DNA complex formation. Genes Dev 8: 995-1006.

Liu WL, Coleman RA, Ma E, Grob P, Yang JL, Zhang Y, Dailey G, Nogales E, Tjian R. 2009. Structures of three distinct activator-TFIID complexes. Genes Dev 23: 1510-1521.

Massari ME, Grant PA, Pray-Grant MG, Berger SL, Workman JL, Murre C. 1999. A conserved motif present in a class of helixloop-helix proteins activates transcription by direct recruitment of the SAGA complex. Mol Cell 4: 63-73.

Mengus G, Fadloun A, Kobi D, Thibault C, Perletti L, Michel I, Davidson I. 2005. TAF4 inactivation in embryonic fibroblasts activates TGF $\beta$ signalling and autocrine growth. EMBO I 24: 2753-2767.

Mizzen CA, Yang XJ, Kokubo T, Brownell JE, Bannister AJ, Owen-Hughes T, Workman J, Wang L, Berger SL, Kouzarides $\mathrm{T}$, et al. 1996. The TAF(II)250 subunit of TFIID has histone acetyltransferase activity. Cell 87: 1261-1270.

Naar AM, Lemon BD, Tjian R. 2001. Transcriptional coactivator complexes. Annu Rev Biochem 70: 475-501.

Oelgeschlager T, Tao Y, Kang YK, Roeder RG. 1998. Transcription activation via enhanced preinitiation complex assembly in a human cell-free system lacking TAFIIs. Mol Cell 1: 925931.

Plevin MJ, Zhang J, Guo C, Roeder RG, Ikura M. 2006. The acute myeloid leukemia fusion protein AML1-ETO targets E proteins via a paired amphipathic helix-like TBP-associated factor homology domain. Proc Natl Acad Sci 103: 1024210247.

Prabhu S, Ignatova A, Park ST, Sun XH. 1997. Regulation of the expression of cyclin-dependent kinase inhibitor p21 by E2A and Id proteins. Mol Cell Biol 17: 5888-5896.

Pugh BF, Tjian R. 1990. Mechanism of transcriptional activation by Sp1: Evidence for coactivators. Cell 61: 1187-1197.

Quong MW, Romanow WI, Murre C. 2002. E protein function in lymphocyte development. Annu Rev Immunol 20: 301-322.

Roeder RG. 1996. The role of general initiation factors in transcription by RNA polymerase II. Trends Biochem Sci 21: 327-335.

Roeder RG. 2005. Transcriptional regulation and the role of diverse coactivators in animal cells. FEBS Lett 579: 909-915.

Saluja D, Vassallo MF, Tanese N. 1998. Distinct subdomains of human TAFII130 are required for interactions with glutaminerich transcriptional activators. Mol Cell Biol 18: 5734-5743. 
Sawadogo M, Roeder RG. 1985. Interaction of a gene-specific transcription factor with the adenovirus major late promoter upstream of the TATA box region. Cell 43: 165-175.

Seidel MG, Look AT. 2001. E2A-HLF usurps control of evolutionarily conserved survival pathways. Oncogene 20: 57185725.

Thomas MC, Chiang CM. 2006. The general transcription machinery and general cofactors. Crit Rev Biochem Mol Biol 41: 105-178.

Trapnell C, Pachter L, Salzberg SL. 2009. TopHat: Discovering splice junctions with RNA-Seq. Bioinformatics 25: 11051111.

Trapnell C, Williams BA, Pertea G, Mortazavi A, Kwan G, van Baren MJ, Salzberg SL, Wold BJ, Pachter L. 2010. Transcript assembly and quantification by RNA-Seq reveals unannotated transcripts and isoform switching during cell differentiation. Nat Biotechnol 28: 511-515.

Wang X, Truckses DM, Takada S, Matsumura T, Tanese N, Jacobson RH. 2007. Conserved region I of human coactivator TAF4 binds to a short hydrophobic motif present in transcriptional regulators. Proc Natl Acad Sci 104: 7839-7844.

Wright KJ, Tjian R. 2009. Wnt signaling targets ETO coactivation domain of TAF4/TFIID in vivo. Proc Natl Acad Sci 106: 55-60.

Wright KJ, Marr MT 2nd, Tjian R. 2006. TAF4 nucleates a core subcomplex of TFIID and mediates activated transcription from a TATA-less promoter. Proc Natl Acad Sci 103: 1234712352.

Zhang J, Kalkum M, Yamamura S, Chait BT, Roeder RG. 2004. E protein silencing by the leukemogenic AML1-ETO fusion protein. Science 305: 1286-1289. 


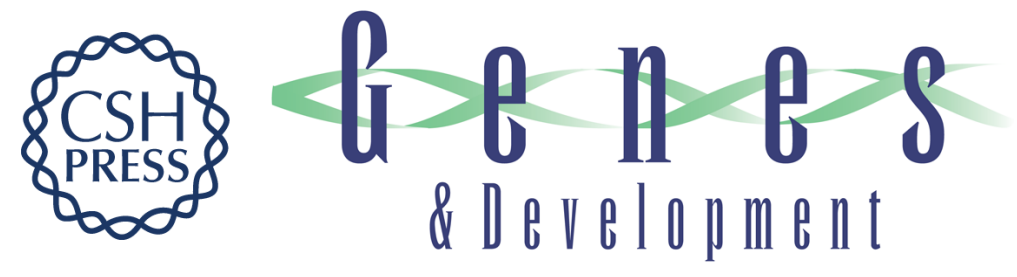

\section{A TAF4 coactivator function for E proteins that involves enhanced TFIID binding}

Wei-Yi Chen, Jinsong Zhang, Huimin Geng, et al.

Genes Dev. 2013, 27:

Access the most recent version at doi:10.1101/gad.216192.113

Supplemental http://genesdev.cshlp.org/content/suppl/2013/07/19/27.14.1596.DC1
Material

References This article cites 45 articles, 17 of which can be accessed free at:

http://genesdev.cshlp.org/content/27/14/1596.full.html\#ref-list-1

License

Email Alerting

Receive free email alerts when new articles cite this article - sign up in the box at the top

Service

right corner of the article or click here.

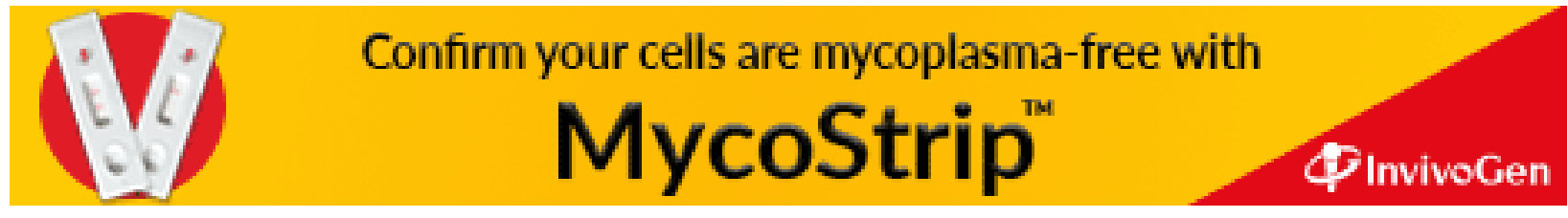

